\title{
Relação Pai-Filha e Transtornos Alimentares: Revisando a Produção Científica*
}

\author{
Manoel Antônio dos Santos** (D) \& Lilian Regiane de Souza Costa-Dalpino (1) \\ ${ }^{1}$ Universidade de São Paulo,São Paulo, SP, Brasil
}

\begin{abstract}
RESUMO - Investigou-se a produção científica dedicada ao estudo da relação entre pai e filha com transtornos alimentares. Foi realizada uma revisão integrativa nas bases PsycINFO, CINAHL, PubMed e LILACS, no período de janeiro de 2000 a junho de 2014. Foram selecionados 27 artigos, a maioria proveniente de países europeus e constituída de estudos quantitativos. A vinculação pouco segura com a figura paterna e o relacionamento emocionalmente distante entre pai e filha foram aspectos recorrentes nas publicações selecionadas. Os estudos apontaram a importância de se investigar e assegurar a participação do pai no tratamento. Porém, nenhum dos artigos revisados investigou o envolvimento paterno no contexto da assistência. Destaca-se a necessidade de investir em estudos qualitativos e no contexto brasileiro.
\end{abstract}

PALAVRAS-CHAVE: relação pai-filha, transtornos alimentares, pai, relações familiares

\section{Father-daughter relationship and eating disorders: a review of the scientific production}

\begin{abstract}
We investigated the scientific production dedicated to the analysis of the relationship between daughters with eating disorders and their fathers. An integrative review was performed on PsycINFO, CINAHL, PubMed and LILACS databases. We reviewed studies published between January 2000 and June 2014. Twenty-seven studies were included in the review. Most were from European countries and were comprised of quantitative studies. An unstable relationship with the father figure and an emotionally distant relationship between father and daughter were recurring themes in the selected publications. Studies showed the importance of investigating and ensuring the participation of fathers in the treatment of eating disorders, but none of them investigated father involvement in terms of assistance. Further research is needed in order to explore qualitative studies in the Brazilian context.
\end{abstract}

KEYWORDS: father-daughter relation, eating disorders, father, family relations, review

Atualmente, as queixas apresentadas pelos pacientes que procuram por atendimento psicológico estão cada vez mais distantes dos quadros clássicos de psiconeuroses descritos por Freud (Birman, 2007; Zimerman, 1999). Na clínica contemporânea, observa-se uma demanda crescente de pacientes com funcionamentos limítrofes e características psicóticas. Gaspar (2005) relata que, nessa "nova clínica", é recorrente a presença de pacientes com queixas associadas à insatisfação com a imagem corporal, como ocorre nos Transtornos Alimentares (TAs).
Birman (2007) chama a atenção para a disseminação das compulsões como parte constitutiva das subjetividades contemporâneas. Isso reflete o fato de que as perturbações psíquicas se condensam cada vez mais nos registros do corpo, da ação e das intensidades (Birman, 2006). Com a exacerbação de aspectos culturais que valorizam medidas antropométricas exíguas, corpos esquálidos e o culto à beleza focalizada apenas na aparência física, os TAs começaram a ganhar maior destaque na sociedade (Santos, Garcia, \& Santos, 2015; Valdanha, Scorsolini-Comin, Peres, \& Santos 2013).

\footnotetext{
* Apoio: Fundação de Amparo à Pesquisa do Estado de São Paulo - FAPESP, mediante bolsa de mestrado (processo número 2012/152777-5) concedida à primeira autora e Conselho Nacional de Desenvolvimento Científico e Tecnológico (CNPq), por meio de Bolsa de Produtividade em Pesquisa nível $1 \mathrm{~A}$ concedida ao segundo autor.

** E-mail: masantos@ffclrp.usp.br

- Submetido: 08/01/2016; Revisado: 14/06/2016; Aceito: 12/08/2016
} 
Os TAs configuram quadros psicopatológicos caracterizados por graves perturbações do comportamento alimentar, que comprometem drasticamente o processo adaptativo e a qualidade de vida da pessoa acometida (American Psychiatric Association [APA], 2013). Os altos índices de incidência e mortalidade associados a esses transtornos evidenciam a necessidade de receberem maior atenção das políticas públicas de saúde (Smink, Van Hoeken, \& Hoek, 2013).

Na quinta edição do Manual Estatístico e Diagnóstico dos Transtornos Mentais (DSM-5; APA, 2013), além dos quadros psicopatológicos abarcados na quarta versão, Anorexia Nervosa (AN), Bulimia Nervosa (BN) e Transtorno Alimentar Não Especificado (TANE) foram incluídos como TAs, além de mais quatro quadros: Transtorno da Compulsão Alimentar Periódica, Transtorno de Consumo Alimentar Evitativo/Restritivo, Pica e Transtorno da Ruminação. Dentre eles, a AN e a BN figuram como os transtornos mais recorrentes. Ambas envolvem a preocupação excessiva com o peso e forma corporal, que leva à utilização de estratégias inapropriadas para evitar o ganho de peso (APA, 2013).

A BN é caracterizada por recorrente descontrole sobre o comportamento alimentar, que provoca episódios de comer compulsivo, seguidos de atitudes compensatórias para evitar o ganho de peso, como vômito autoinduzido e abuso de laxantes. A AN designa um quadro clínico marcado por intensa restrição da alimentação, acompanhada pelo medo excessivo do ganho de peso e intensa distorção da imagem corporal. O comportamento restritivo persiste mesmo com peso inferior ao mínimo esperado para idade e altura e frequentemente acarreta um grau de desnutrição severa e incompatível com a vida. A anorexia se divide em dois subtipos: restritivo e bulímico. No segundo, além das restrições, também são observados episódios de purgação (APA, 2013).

Embora sejam psicopatologias já descritas no passado, foi só no decorrer das últimas duas décadas que elas passaram a ter maior expressão no contexto social, o que, muitas vezes, dá a impressão de que seriam "novas psicopatologias". Sua maior presença, ao lado de outras que também não eram tão percebidas no passado, é entendida como sinal de maior percepção social, decorrente da disseminação de conhecimentos sobre esses transtornos na sociedade ou de possíveis mudanças na formação da estrutura de personalidade do indivíduo contemporâneo.

Para alguns autores, as raízes dessas mudanças podem ser buscadas nas modificações que as relações familiares sofreram e continuam sofrendo com o passar dos anos, face às novas formas de subjetivação (Barbieri, 2013; Birman, 2007). A família é o primeiro grupo responsável por suprir as necessidades básicas do bebê, entre elas, a alimentação. $\mathrm{Na}$ espécie humana, o ato de alimentar é permeado por simbolismos e influências psicológicas e culturais (Moura, Santos, \& Ribeiro, 2015). Desse modo, apesar de a família ser uma instituição universal, o modo como ela se organiza diferencia-se bastante entre as sociedades, o que traz repercussões também distintas para a organização da personalidade (Boarini, 2003).

No contexto dos TAs, o grupo familiar tem sido definido pela literatura da área como um dos fatores etiológicos responsáveis pelo desenvolvimento dos sintomas. As famílias dos indivíduos acometidos por essas psicopatologias tendem a apresentar padrões de funcionamento característicos, que colaboram com o surgimento e manutenção do problema. São famílias que enfrentam dificuldades de estabelecer limites entre seus membros e entre os diversos subsistemas: conjugal, parental e fraterno. Consequentemente, percebe-se confusão entre os papéis que cada um assume na dinâmica das relações familiares, o que prejudica a constituição da identidade de todos os envolvidos, especialmente daqueles membros que se encontram nas etapas iniciais do desenvolvimento (Oliveira \& Santos, 2006).

Apesar de os estudos indicarem que a família, como um todo, vivencia prejuízos na busca pela individualidade, as pesquisas claramente privilegiam a investigação da relação mãe-filha (Miranda, 2010). As justificativas dos estudos para a maior ênfase dedicada à figura materna estão amparadas na evidência de que a manifestação dos TAs estaria relacionada a vivências emocionais muito precoces, quando o vínculo entre mãe e bebê ainda é muito estreito e marcado pela indiferenciação psíquica. Valdanha et al. (2013) descrevem o sintoma anoréxico como uma tentativa de realizar o desejo inconsciente de se separar da figura materna, sentida como extremamente invasiva e controladora. Desse modo, a recusa em se alimentar funcionaria como uma via defensiva de proteção do self contra o outro - representado pelo alimento - percebido como nocivo e intrusivo (Fava \& Peres, 2011; Miranda, 2010).

A dinâmica interpsíquica problemática entre mãe e filha com TAs (Moura et al., 2015), somada ao tradicional afastamento do pai no cuidado dos filhos (Vieira \& Nascimento, 2014), contribuíram para que a figura paterna permanecesse em um segundo plano nos estudos da área e também no cotidiano da assistência (Souza \& Santos, 2007). Entretanto, há, na literatura, farto conhecimento consolidado que evidencia que o pai também assume importantes funções para a construção da personalidade e da identidade dos filhos, desde o seu nascimento (Zimerman, 1999).

As mudanças socioeconômicas recentes vivenciadas pela sociedade trouxeram à tona a necessidade de maior envolvimento paterno no cuidado dos filhos. A ampla inserção da mulher no mercado de trabalho e as novas configurações familiares foram os fatores que mais influenciaram que o pai começasse a ser cobrado a assumir novas funções na relação com os filhos (Vieira \& Nascimento, 2014). Em decorrência desse novo cenário, as forças vivas da sociedade, como o aparelho educacional, as representações midiáticas e as próprias mulheres emancipadas, passaram a reafirmar a importância da participação do pai no desenvolvimento da prole, ao mesmo tempo em que o Estado começou a implementar políticas públicas que buscam evitar a negligência paterna. 
Em resposta às profundas modificações sociais dos últimos tempos, a investigação científica da área da saúde tem se preocupado cada vez mais com a abordagem da figura paterna. Porém, esse interesse aumentado ainda é tímido (Henn \& Sifuentes, 2012), pois, nas pesquisas sobre o desenvolvimento infantil, o pai ainda continua ausente. Oliveira e Silva (2011), em uma revisão da literatura, encontraram que somente a partir do ano de 2004 os estudos no campo da psicologia incrementaram sua preocupação com a investigação da figura paterna. Nas décadas anteriores, o pai aparecia apenas como mero coadjuvante no cuidado dos filhos.

Partindo da constatação de que persiste uma abordagem incipiente da figura paterna no âmbito da assistência à saúde, o presente estudo teve como objetivo investigar a produção científica dedicada ao estudo da relação entre pai e filha com diagnóstico de TAs.

\section{MÉTODO}

Foi realizada uma revisão integrativa da literatura. Essa estratégia é um tipo de revisão sistemática que compreende uma investigação ampla da literatura, que possibilita sintetizar os estudos mais qualificados, conhecer os métodos utilizados e realçar os achados mais significativos sobre o assunto de interesse (Whitemore \& Knafl, 2005). Além disso, por meio dos dados encontrados, é possível conhecer os temas que mais demandam atenção dos pesquisadores e as lacunas encontradas em um campo específico do conhecimento (Santos \& Santos, 2015).

Revisões integrativas permitem a inclusão e a análise crítica de estudos de diferentes metodologias - tanto empíricos, como teóricos -, resultados de outras revisões e questões metodológicas, visando à compreensão aprofundada de um fenômeno. Os resultados possibilitam qualificar práticas baseadas em evidências fortes, desde que o rigor do método seja respeitado (Whitemore \& Knafl, 2005). Desse modo, a revisão integrativa constitui-se como um instrumento para subsidiar o desenvolvimento e a elucidação de novos estudos empíricos (Valdanha et al., 2013).

A presente revisão buscou evidenciar o número de trabalhos publicados na área, o perfil dos estudos realizados e suas principais contribuições para a compreensão das relações familiares (particularmente, a relação pai-filha) de mulheres acometidas por psicopatologias como AN e BN. A pergunta que norteou a revisão pode ser delineada da seguinte maneira: "O que se sabe, no atual estágio do conhecimento, sobre a relação pai-filha no contexto dos transtornos alimentares?".

Com o intuito de garantir a abrangência nacional e internacional da pesquisa, foram selecionadas as seguintes bases indexadoras: PubMed, CINAHL, PsycINFO e LILACS. As três primeiras são bases internacionais, consagradas na Medicina, na Enfermagem e na Psicologia; e a última é uma base genérica, que colige a produção científica dos países latino-americanos e do Caribe. Para operacionalizar a busca, foram utilizados os seguintes Descritores em Ciências da Saúde (DeCS): transtornos alimentares (eating disorders), pais progenitores do sexo masculino (fathers), relações pai-filho (father-child relations) e relações familiares (family relations), em várias combinações.

Para a seleção dos estudos que compõem o corpus deste estudo, utilizaram-se como critérios de inclusão: abordar o tema alvo da presente revisão, estar disponível na íntegra nas bases consultadas por meio da RedeUSP, ter sido veiculado na língua portuguesa, inglesa ou espanhola e ter sido publicado no período de janeiro de 2000 a junho de 2014. Foram excluídos estudos não publicados em formato de artigo, tais como dissertações, teses, livros, capítulos, monografias, cartas e editoriais. A partir da leitura e análise dos resumos disponibilizados, foram recuperados na íntegra apenas os artigos que preenchiam os critérios de elegibilidade definidos no presente estudo.

A consulta às bases foi operacionalizada em quatro etapas. Primeiramente, foi realizada a busca nas bases com a combinação dos descritores escolhidos. Em seguida, os resumos dos estudos encontrados foram lidos e analisados a partir dos critérios de inclusão/exclusão estabelecidos, sendo excluídos aqueles que não correspondiam ao escopo da pesquisa. Na terceira etapa, foram excluídos artigos repetidos, isto é, disponibilizados em mais de uma base indexadora. Por fim, foram analisadas na íntegra as publicações selecionadas, que constituíram o corpus deste estudo (Valdanha et al., 2013).

Os artigos recuperados na íntegra foram submetidos a uma leitura analítica, realizada de modo independente, por dois pesquisadores com experiência na aplicação do procedimento. Para atender ao objetivo proposto, foi delineado um formulário para extrair os dados dos artigos, composto pelas principais variáveis de interesse para o presente estudo.

A apreciação do material envolveu, inicialmente, a identificação das seguintes dimensões: ano de publicação, país de origem do estudo, participantes, objetivos e referencial teórico. Para assegurar a fidedignidade da classificação, os resultados que obtiveram consenso entre os dois avaliadores foram aceitos automaticamente. Já as análises que se revelaram discordantes foram discutidas caso a caso, até que se chegasse a um comum acordo (Valdanha et al., 2013).

A partir da análise criteriosa dos resultados encontrados, os artigos revisados foram divididos em cinco núcleos temáticos, de acordo com os passos recomendados por Minayo (2007), que permitiram organizar a apresentação dos dados. Os núcleos temáticos encontrados foram: (1) Aspectos da vinculação e apego entre pai e filha; (2) Traços de personalidade e estilo parental; (3) Aspectos psicopatológicos do pai; (4) Perspectiva do pai sobre o funcionamento familiar e (5) Participação do pai no tratamento. Os achados foram discutidos a partir dos temas encontrados. 


\section{RESULTADOS E DISCUSSÃO}

As quatro bases de dados forneceram, preliminarmente, um total de 745 referências. Após aplicação dos critérios de inclusão/exclusão, foram selecionados 74 estudos. Ao serem descartados os artigos repetidos em mais de uma base indexadora, restaram 27 estudos, que constituíram o corpus dessa revisão. A Figura 1 condensa as informações sobre o número de artigos encontrados nas bases consultadas, nas quatro etapas de refinamento da busca bibliográfica. PsycINFO foi a base na qual foi encontrado o maior número de publicações consideradas pertinentes para esta revisão.

Em todas as bases utilizadas, a maior parte dos artigos localizados respondeu à combinação "eating disorders" e "family relations". Com essa combinação, foram encontrados estudos que abordavam variados aspectos das relações familiares no contexto dos TAs. A maioria deles referia-se a particularidades da relação mãe-filha ou à família como um todo, sem estabelecer diferenciações entre os membros. Foram escolhidos apenas os artigos que, de alguma forma, adicionavam dados concernentes ao pai, mesmo que também abordassem a figura materna.

A Tabela 1 detalha o número de referências encontradas e selecionadas nas bases indexadoras, em função da combinação dos DeCs utilizados na busca. A combinação dos descritores "eating disorders and family relations" mostrou-se a mais proveitosa, pois possibilitou recuperar 490 referências, dentre as quais foram recuperadas 21 , que preenchiam os critérios de inclusão.

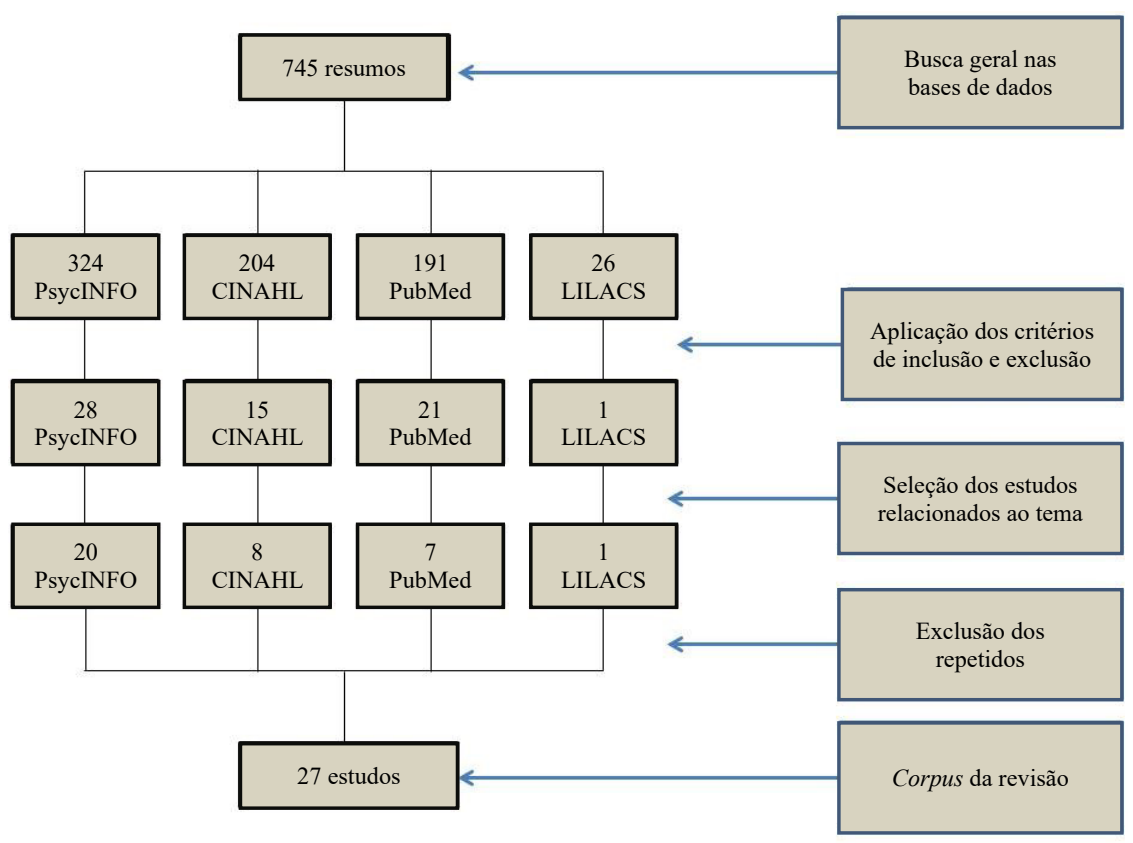

Figura 1. Fluxograma da revisão integrativa.

Tabela 1.

Número de referências encontradas (E) e selecionadas (S) nas bases indexadoras, a partir da combinação dos Descritores em Ciências da Saúde (DeCs) utilizados.

\begin{tabular}{|c|c|c|c|c|c|c|c|c|}
\hline \multirow{2}{*}{ Descritores } & \multicolumn{2}{|c|}{ PsycINFO } & \multicolumn{2}{|c|}{ CINAHL } & \multicolumn{2}{|c|}{ PubMed } & \multicolumn{2}{|c|}{ LILACS } \\
\hline & $\mathbf{E}$ & $\mathbf{S}$ & $\mathbf{E}$ & $\mathbf{S}$ & $\mathbf{E}$ & $\mathbf{S}$ & $\mathbf{E}$ & $\mathbf{S}$ \\
\hline Eating disorders and father-child relations & 16 & 8 & 4 & 3 & 18 & 8 & 1 & - \\
\hline Eating disorders and family relations & 186 & 5 & 196 & 9 & 108 & 7 & 14 & - \\
\hline Eating disorders and fathers & 122 & 15 & 4 & 3 & 65 & 7 & 11 & 1 \\
\hline Total & 324 & 28 & 204 & 15 & 191 & 21 & 26 & 1 \\
\hline
\end{tabular}


Entre os estudos selecionados, houve predomínio de artigos publicados entre os anos de 2009 e 2014, totalizando $59,2 \%$ da amostra. Esse percentual sugere que o interesse científico pelo tema tem sido crescente. No que se refere aos locais de publicação, a maior parcela dos artigos é oriunda do Reino Unido $(\mathrm{n}=7)$ e dos Estados Unidos ( $\mathrm{n}=5)$, seguidos de três estudos italianos, dois chilenos e dois poloneses. Israel, Nova Zelândia, Portugal, Alemanha, Canadá, Suécia, Espanha e Bélgica são países de origem dos autores de uma publicação selecionada. Não foi localizado estudo brasileiro dedicado ao tema investigado.

Como se pode observar na Tabela 2, a maioria dos estudos selecionados $(n=13)$ teve como participantes apenas as filhas. Foram investigadas as características da figura paterna a partir da perspectiva das filhas que desenvolveram os TAs. Três estudos tiveram como participantes a díade formada por pai e filha (Benninghoven, Tetsch, Kunzendorf, \& Jantschek, 2007; Dixon, Gill, \& Adair, 2003; Hooper \& Dallos, 2012). Nenhum estudo encontrado trabalhou com a figura paterna isoladamente e apenas um incluiu irmãos como participantes da pesquisa (Enten \& Golan, 2009).

Estudos com métodos quantitativos foram preponderantes e analisaram como aspectos relacionados à figura paterna e à maneira como os pais se relacionam com suas filhas podem influenciar o desenvolvimento dos sintomas de TAs. Apenas quatro dos 27 estudos que compõem a amostra adotaram uma perspectiva qualitativa (Elliott, 2010; Guzmán, Hitelman, \& Kaplan, 2009; Hooper \& Dallos, 2012; Nodin \& Leal, 2005). Esses estudos são exploratórios e investigaram aspectos da relação pai-filha no contexto dos TAs. Três deles adotaram como referencial teórico a psicanálise (Elliott, 2010; Guzmán et al., 2009; Nodin \& Leal, 2005). O estudo de Hooper e Dallos (2012) utilizou o referencial fenomenológico.

Apesar de não ter sido especificado previamente quais TAs seriam investigados no presente estudo, foram encontradas apenas publicações que investigaram pacientes com $\mathrm{AN}$ e $\mathrm{BN}$ ou sintomas relacionados a essas psicopatologias, o que pode ser justificado pela maior prevalência desses quadros entre os TAs, bem como pelo fato de a maioria dos estudos ter sido publicada antes da inclusão das novas categorias diagnósticas no DSM-5 (APA, 2013).

Diante dos poucos estudos encontrados que abordavam especificamente o pai de filhas que desenvolveram psicopatologias do comportamento alimentar como os TAs, foi necessário operar um refinamento da busca em estudos que envolviam a família como um todo, especialmente a tríade pai-mãe-filha. Somente com essa busca mais aprofundada foi possível identificar maior contingente de estudos no cenário da investigação científica que relaciona a figura paterna aos TAs. Os dados foram agrupados e discutidos a partir dos cinco núcleos temáticos encontrados, que serão apresentados e discutidos a seguir.

Tabela 2.

Caracterização dos artigos recuperados segundo autores, ano, país da publicação, população estudada e delineamento do estudo ( $n=27)$.

\begin{tabular}{|c|c|c|c|c|}
\hline Autores & Ano & País & População estudada & Delineamento do estudo \\
\hline $\begin{array}{l}\text { Pilecki, Józefik, } \\
\& \text { Salapa }\end{array}$ & 2014 & Polônia & $\begin{array}{l}233 \text { tríades compostas por pai, mãe e filha, divididas em três grupos: (1) } \\
45 \text { filhas com AN do tipo restritivo (AN-R), } 18 \text { com AN do tipo bulímico } \\
\text { (AN-B) e } 25 \text { com BN; (2) } 36 \text { mulheres com transtornos depressivos; (3) } 85 \\
\text { filhas sem diagnóstico psiquiátrico. }\end{array}$ & $\begin{array}{l}\text { Não experimental, } \\
\text { descritivo e correlacional }\end{array}$ \\
\hline Horesh et al. & 2014 & $\begin{array}{l}\text { Reino } \\
\text { Unido }\end{array}$ & $\begin{array}{l}53 \text { mulheres com diagnóstico de TAs (não foram especificadas quantas } \\
\text { tinham recebido cada diagnóstico) e dois grupos controle: (1) } 26 \\
\text { participantes com outros transtornos psiquiátricos e (2) } 60 \text { participantes } \\
\text { saudáveis. }\end{array}$ & $\begin{array}{l}\text { Não experimental, } \\
\text { descritivo e correlacional }\end{array}$ \\
\hline Amianto et al. & 2013 & Itália & $\begin{array}{l}108 \text { mães e } 104 \text { pais de pacientes com diagnóstico de TAs ( } 63 \text { filhas com } \\
\text { AN; } 31 \text { com BN e } 61 \text { com TANE. }\end{array}$ & $\begin{array}{l}\text { Não experimental, } \\
\text { descritivo e correlacional }\end{array}$ \\
\hline $\begin{array}{l}\text { Pilecki \& } \\
\text { Józefik }\end{array}$ & 2013 & Polônia & $\begin{array}{l}233 \text { tríades compostas por pai, mãe e filhas, divididas em três grupos: } \\
\text { (1) } 54 \text { filhas com AN-R; } 22 \text { com AN-B e } 36 \text { com BN; (2) } 36 \text { filhas com } \\
\text { transtornos depressivos; (3) } 85 \text { filhas sem diagnóstico psiquiátrico. }\end{array}$ & $\begin{array}{l}\text { Não experimental, } \\
\text { descritivo e correlacional }\end{array}$ \\
\hline Martín et al. & 2013 & Espanha & $\begin{array}{l}113 \text { pacientes com diagnóstico de TAs (não foi especificado quantas com } \\
\text { cada diagnóstico), } 111 \text { mães e } 70 \text { pais. }\end{array}$ & $\begin{array}{l}\text { Não experimental, } \\
\text { descritivo e correlacional }\end{array}$ \\
\hline $\begin{array}{l}\text { Hooper \& } \\
\text { Dallos }\end{array}$ & 2012 & $\begin{array}{l}\text { Reino } \\
\text { Unido }\end{array}$ & Três díades pai-filha (filhas com AN). & $\begin{array}{l}\text { Qualitativo, exploratório } \\
\text { e descritivo }\end{array}$ \\
\hline $\begin{array}{l}\text { Haycraft \& } \\
\text { Blissett. }\end{array}$ & 2012 & $\begin{array}{l}\text { Reino } \\
\text { Unido }\end{array}$ & 48 díades compostas por mãe e pai de crianças sem diagnóstico de TAs. & $\begin{array}{l}\text { Não experimental, } \\
\text { descritivo e correlacional }\end{array}$ \\
\hline Goossens et al. & 2012 & Bélgica & 601 pré-adolescentes sem diagnóstico de TAs. & $\begin{array}{l}\text { Quase-experimental, } \\
\text { correlacional }\end{array}$ \\
\hline Lam \& McHale & 2012 & $\begin{array}{l}\text { Estados } \\
\text { Unidos }\end{array}$ & 201 famílias (pai, mãe e dois filhos adolescentes sem diagnóstico de TAs). & $\begin{array}{l}\text { Quase-experimental, } \\
\text { longitudinal. }\end{array}$ \\
\hline $\begin{array}{l}\text { Pace, Cacioppo, } \\
\& \text { Schimmenti }\end{array}$ & 2012 & Itália & 233 estudantes do sexo feminino sem diagnóstico de TAs. & $\begin{array}{l}\text { Não experimental, } \\
\text { descritivo e correlacional }\end{array}$ \\
\hline Back & 2011 & Suécia & $\begin{array}{l}80 \text { estudantes universitários sem diagnóstico de TAs (média de idade de } 18 \\
\text { anos, sendo } 45 \text { homens e } 35 \text { mulheres). }\end{array}$ & $\begin{array}{l}\text { Não experimental, } \\
\text { descritivo e correlacional }\end{array}$ \\
\hline
\end{tabular}


Tabela 2 .

\begin{tabular}{|c|c|c|c|c|}
\hline Autores & Ano & País & População estudada & Delineamento do estudo \\
\hline Elliott & 2010 & $\begin{array}{l}\text { Estados } \\
\text { Unidos }\end{array}$ & 11 pacientes com diagnóstico de $\mathrm{AN}$. & $\begin{array}{l}\text { Qualitativo, descritivo e } \\
\text { exploratório }\end{array}$ \\
\hline $\begin{array}{l}\text { Fassino, } \\
\text { Amianto, \& } \\
\text { Abbate-Daga }\end{array}$ & 2009 & Itália & $\begin{array}{l}105 \text { mulheres com TAs ( } 38 \text { com AN-R; } 30 \text { com AN-B e } 37 \text { com BN), } 87 \\
\text { pais e } 97 \text { mães. Grupo controle constituído por } 54 \text { mulheres, } 50 \text { pais e } 54 \\
\text { mães. }\end{array}$ & $\begin{array}{l}\text { Não experimental, } \\
\text { descritivo e correlacional }\end{array}$ \\
\hline Enten \& Golan & 2009 & Israel & $\begin{array}{l}53 \text { famílias, compostas por: } 53 \text { filhas com TAs ( } 32 \text { com AN, } 18 \text { com BN, } 3 \\
\text { com TANE), } 52 \text { mães, } 51 \text { pais e } 20 \text { irmãos. }\end{array}$ & $\begin{array}{l}\text { Não experimental, } \\
\text { descritivo e correlacional }\end{array}$ \\
\hline $\begin{array}{l}\text { McEwen \& } \\
\text { Flouri }\end{array}$ & 2009 & $\begin{array}{l}\text { Reino } \\
\text { Unido }\end{array}$ & 203 estudantes do sexo feminino sem diagnóstico de TAs. & $\begin{array}{l}\text { Não experimental, } \\
\text { descritivo e correlacional }\end{array}$ \\
\hline $\begin{array}{l}\text { Guzmán, } \\
\text { Hitelman, \& } \\
\text { Kaplan }\end{array}$ & 2009 & Chile & Uma paciente com diagnóstico de AN. & $\begin{array}{l}\text { Qualitativo, descritivo e } \\
\text { exploratório }\end{array}$ \\
\hline Cruzat et al. & 2008 & Chile & 296 estudantes do ensino médio sem diagnóstico de TAs. & $\begin{array}{l}\text { Não experimental, } \\
\text { descrito e correlacional }\end{array}$ \\
\hline $\begin{array}{l}\text { Benninghoven, } \\
\text { Kunzendorf, } \\
\text { \& Jantschek }\end{array}$ & 2007 & Alemanha & 42 díades compostas por pai e filha ( 27 filhas com $\mathrm{AN}$ e 15 com $\mathrm{BN}$ ). & $\begin{array}{l}\text { Não experimental, } \\
\text { descritivo e correlacional }\end{array}$ \\
\hline $\begin{array}{l}\text { Alda, Espina, } \\
\& \text { Ortego }\end{array}$ & 2006 & Espanha & $\begin{array}{l}190 \text { tríades compostas por pai, mãe e filha. } 32 \text { com filhas com AN-R, } 31 \\
\text { com AN-B, } 37 \text { com BN e } 90 \text { sem diagnóstico de TAs (grupo controle). }\end{array}$ & $\begin{array}{l}\text { Quase-experimental, } \\
\text { descritivo e correlacional }\end{array}$ \\
\hline $\begin{array}{l}\text { Jones, Leung, } \\
\& \text { Harris }\end{array}$ & 2006 & $\begin{array}{l}\text { Reino } \\
\text { Unido }\end{array}$ & $\begin{array}{l}66 \text { mulheres com sintomas de } \mathrm{AN} \text { e BN (não foram especificadas quantas } \\
\text { tinham recebido cada diagnóstico) e um grupo controle com } 50 \text { mulheres } \\
\text { sem diagnóstico de TAs. }\end{array}$ & $\begin{array}{l}\text { Não experimental, } \\
\text { descritivo e correlacional }\end{array}$ \\
\hline Nodin \& Leal & 2005 & Portugal & Nove mulheres com diagnóstico de AN. & $\begin{array}{l}\text { Qualitativo, descritivo e } \\
\text { exploratório }\end{array}$ \\
\hline Dancyger et al. & 2005 & $\begin{array}{l}\text { Estados } \\
\text { Unidos }\end{array}$ & $\begin{array}{l}126 \text { mulheres com diagnóstico de TAs ( } 24 \text { com AN-R, } 23 \text { com AN-B, } 41 \\
\text { com BN, } 38 \text { com TANE), } 118 \text { mães e } 96 \text { pais. }\end{array}$ & $\begin{array}{l}\text { Não experimental, } \\
\text { descritivo e correlacional }\end{array}$ \\
\hline $\begin{array}{l}\text { Jones, Harris, } \\
\text { \& Leung }\end{array}$ & 2005 & $\begin{array}{l}\text { Reino } \\
\text { Unido }\end{array}$ & $\begin{array}{l}66 \text { mulheres com diagnóstico de } \mathrm{AN} \text { e } \mathrm{BN} \text { (sem especificar quantas tinham } \\
\text { recebido esses diagnósticos). }\end{array}$ & $\begin{array}{l}\text { Não experimental, } \\
\text { descritivo e correlacional }\end{array}$ \\
\hline $\begin{array}{l}\text { Gutzwiller, } \\
\text { Oliver, \& Katz }\end{array}$ & 2003 & $\begin{array}{l}\text { Estados } \\
\text { Unidos }\end{array}$ & $\begin{array}{l}306 \text { estudantes do curso de graduação em Psicologia sem diagnóstico de } \\
\text { TAs. }\end{array}$ & $\begin{array}{l}\text { Não exprimental, } \\
\text { descritivo e correlacional }\end{array}$ \\
\hline $\begin{array}{l}\text { Dixon, Gill, \& } \\
\text { Adair }\end{array}$ & 2003 & $\begin{array}{l}\text { Nova } \\
\text { Zelândia }\end{array}$ & 50 díades pai-filha com filhas sem diagnóstico de TAs. & $\begin{array}{l}\text { Não experimental, } \\
\text { descritivo e correlacional }\end{array}$ \\
\hline $\begin{array}{l}\text { Botta \& } \\
\text { Dumlao }\end{array}$ & 2002 & $\begin{array}{l}\text { Estados } \\
\text { Unidos }\end{array}$ & 210 mulheres universitárias sem diagnóstico de TAs. & $\begin{array}{l}\text { Não experimental, } \\
\text { descritivo e correlacional }\end{array}$ \\
\hline $\begin{array}{l}\text { Rowa, Kerig, \& } \\
\text { Geller }\end{array}$ & 2001 & Canadá & $\begin{array}{l}95 \text { tríades compostas por pai, mãe e filha. } 30 \text { filhas com AN e } 65 \text { filhas sem } \\
\text { diagnóstico de TAs (grupo controle). }\end{array}$ & $\begin{array}{l}\text { Não experimental, } \\
\text { descritivo e correlacional }\end{array}$ \\
\hline
\end{tabular}

\section{Aspectos da Vinculação e Apego entre Pai e Filha}

O modo como é estabelecida a vinculação com as figuras parentais emerge como uma das principais preocupações dos estudos da área. No que se refere à vinculação com a figura paterna, a literatura encontrada é concisa ao afirmar que a existência de um vínculo pouco seguro, distante e com poucas possibilidades de comunicação com o pai está correlacionada positivamente com o desenvolvimento de TAs nas filhas (Back, 2011; Botta \& Dumlao, 2002; Cruzat, Ramírez, Melipillán, \& Marzolo, 2008; Elliot, 2010; Goossens, Braet, Vand Durme, Decaluwé, \& Bosmans, 2012; Hopper \& Dallos, 2012; Lam \& McHale, 2012; Pace, Cacioppo, \& Schimmenti, 2012; Rowa, Kerig, \& Geller, 2001).
Elliot (2010) reportou que o distanciamento entre pai e filha se intensifica com o início da adolescência. Diante do afastamento paterno, Jones, Harris e Leung (2005) e Jones, Leung e Harris (2006) destacam que as filhas temem ser rejeitadas pelo pais. Para Elliott (2010), devido ao receio do abandono, as filhas idealizam a figura paterna, além de enfatizarem as similaridades entre seu temperamento e o dos pais. Segundo a análise da autora, a necessidade de receberem aprovação dos pais as levaria a buscarem assemelhar-se a eles.

Gutzwiller, Oliver e Katz (2003) também encontraram que mulheres com sintomas de TAs (mais especificamente, $\mathrm{AN}$ e BN) apresentam vinculação menos segura com a figura paterna, quando comparadas ao grupo controle. Todavia, são relatados resultados diferenciados: mulheres 
que preenchiam os critérios para o diagnóstico de TAs apresentaram melhor qualidade de vinculação com o pai e menores índices de sintomas depressivos do que as garotas que não preenchiam todos os critérios. Os autores sugerem três explicações plausíveis para esse resultado: diferenças nos diagnósticos, diferenças na cronicidade do quadro e o fato de que os sintomas de TAs podem encobrir os sintomas depressivos e mascarar a percepção adequada do vínculo com a figura paterna. Desse modo, percebe-se a necessidade de mais estudos de natureza qualitativa que aprofundem a compreensão do marcado distanciamento entre pais e filhas.

Além do vínculo pouco seguro, Back (2011), Botta e Dumlao (2002) e Cruzat et al. (2008) acrescentam que a existência de uma vinculação permeada por conflitos entre pai e filha pode contribuir para o desenvolvimento de TAs nas filhas. De acordo com Back (2011), esses conflitos estão relacionados ao medo que as filhas sentem em relação ao pai. Assim, observou-se que a existência de uma comunicação eficaz entre pai-filha pode reduzir as possibilidades do desenvolvimento de sintomas desses transtornos nas filhas.

\section{Traços de Personalidade e Estilo Parental}

Paralelamente à percepção da vinculação pouco segura entre pai e filha, no contexto dos TAs, verifica-se um esforço da literatura em encontrar traços de personalidade e estilos parentais comuns aos pais, que justificariam a preponderância desse padrão vincular com as filhas. Entretanto, os resultados obtidos com a presente revisão apontaram para a dificuldade de se estabelecer um perfil típico da figura paterna no cenário dos TAs. Amianto, Daga, Bertorello e Fassino (2013), por exemplo, ao tentarem dividir os pais de indivíduos com TAs em grupos de traços de personalidade, obtiveram uma distribuição aleatória dos participantes entre os clusters. Os pais se subdividiram em dois perfis de temperamento: explosivo/metódico (49\% da amostra) e independente/metódico (51\% da amostra). Esses dados evidenciam uma heterogeneidade de perfis de personalidade dos pais, embora se possa afirmar que o tipo metódico tenda a se impor.

Apesar da impossibilidade de generalização dos dados, os resultados obtidos com esta revisão apontaram para características predominantes no perfil de personalidade dos pais de pessoas com TAs. Todavia, é importante ressaltar que 13 estudos que compõem o corpus da presente revisão tiveram como participantes apenas as filhas. Isso limita a compreensão da personalidade dos pais e dos estilos parentais, já que esses dados poderiam ser alterados se a visão dos pais sobre si próprios também fosse incorporada, bem como a percepção de outros membros familiares. Além disso, o foco da maior parte dos estudos foi colocado nos pais de mulheres que receberam diagnóstico de AN. Quatro artigos investigaram apenas pacientes com sintomas anoréxicos e não houve qualquer estudo que se deteve apenas nos sintomas bulímicos.
Em relação às características dos progenitores do sexo masculino, correlacionadas positivamente com o desenvolvimento de TAs nas filhas, um dos temas recorrentes nos estudos revisados foi a autoridade paterna. Enten e Golan (2009), Jones et al. (2005, 2006) e McEwen e Flouri (2009) observaram, empiricamente, que a figura de um pai controlador e a superproteção paterna podem ser fatores precipitadores de TAs nas filhas. Os dados de Pace et al. (2012) acrescentam que o medo em relação à figura de autoridade pode aumentar o risco da presença de compulsão alimentar nas filhas. Em contrapartida, Enten e Golan (2009) encontraram que a falta de autoridade paterna também pode ser um fator que influencia o desencadeamento de TAs nas filhas.

Outra característica que a literatura aponta como frequente nos pais de pacientes com TAs é a tendência à esquiva, principalmente nos pais de mulheres diagnosticadas com AN do tipo restritivo (Elliot, 2010; Fassino, Amianto, \& Abbate-Daga, 2009; Hopper \& Dallos, 2012; Horesh, Sommerfeld, Wolf, Zuberi, \& Zalsman, 2014; Nodin \& Leal, 2005). As participantes do estudo de Elliot (2010), 11 mulheres que receberam diagnóstico de $\mathrm{AN}$, identificaram o pai como uma pessoa que estava emocional e fisicamente indisponível. Ao investigarem a relação pai-filha nos TAs e sintomas depressivos das pacientes, Horesh et al. (2014) concluíram que atributos negativos dos pais foram significativamente associados com os transtornos e sintomas depressivos das filhas.

A tendência à esquiva do pai pode justificar o temor de abandono que Jones et al. $(2005,2006)$ encontraram nas filhas com anorexia. Porém, apesar de perceberem o pai como alguém distante afetivamente, as participantes dos estudos de Elliot (2010) e Nodin e Leal (2005) evidenciaram uma percepção idealizada da figura paterna. Segundo análise de Nodin e Leal (2005), os pais de mulheres com AN não teriam assumido a esperada função de interdição ao longo do desenvolvimento psicossexual das filhas. Todavia, como formação reativa, sua figura aparece muito investida afetivamente por elas.

Seguindo essa vertente de análise, Guzmán et al. (2009) concluíram que a anorexia é uma busca concreta pela presença paterna, uma vez que o pai não se mostrou presente em momentos cruciais do desenvolvimento da filha. $\mathrm{O}$ fato de os três estudos mencionados serem de abordagem qualitativa facilitou a compreensão, com maior profundidade, das peculiaridades dos estilos parentais dos pais.

\section{Aspectos Psicopatológicos do Pai}

Hopper e Dallos (2012) chamam a atenção para a necessidade de se investigarem os motivos que levariam ao distanciamento paterno no cuidado das filhas com TAs. Entretanto, foram encontrados poucos estudos que se propuseram a identificar aspectos psicopatológicos do pai que supostamente poderiam influenciar o desenvolvimento e 
a manutenção dos sintomas de TAs nas filhas, principalmente quando são comparados com o número de artigos que investigaram o funcionamento das mães.

Apesar da escassez de publicações sobre o tema investigado, foi possível encontrar dados relevantes sobre o funcionamento psíquico, até mesmo psicopatológico, dos pais cujas filhas desenvolveram TAs. Esses dados podem ser divididos entre aqueles estudos que tiveram como foco de investigação os aspectos psicopatológicos relacionados à percepção da imagem corporal e os que abarcaram a autoestima e sintomas de depressão e ansiedade.

Preocupação com a imagem corporal. Dentre os estudos selecionados na presente revisão, quatro deles se preocuparam em investigar aspectos psicopatológicos relacionados à percepção da imagem corporal dos pais e se esses aspectos influenciam ou não o surgimento dos sintomas dos TAs nas filhas (Benninghoven et al., 2007; Dixon et al., 2003; Haycraft \& Blissett, 2012; Lam \& McHale, 2012). Os resultados encontrados são bastante difusos, porém todos evidenciaram que a preocupação paterna com a própria imagem corporal pode influenciar a maior preocupação das filhas com o seu próprio peso e, consequentemente, pode estar relacionada ao surgimento dos TAs. Além disso, Haycraft e Blissett (2012) acrescentam que a baixa ingestão alimentar do pai pode interferir na restrição da alimentação das filhas.

Mais especificamente, Benninghoven et al. (2007) identificaram que a maioria dos pais de pacientes com TAs conseguia ter uma percepção adequada de seu corpo, porém desejava adquirir massa muscular e perder gordura corporal. O desejo de ter maior massa muscular mostrou-se positivamente correlacionado com o desejo de redução da gordura corporal manifestado pelas filhas com BN. Corroborando esses achados, Dixon et al. (2003) encontraram que, quando o pai acreditava fortemente na importância do cuidado com a alimentação e com o corpo para se manter atraente, sua filha estava mais propensa a induzir vômitos com o intuito de perder peso.

Elliott (2010) e Nodin e Leal (2005) identificaram uma tendência das filhas de se apresentarem como parecidas com o pai, o que foi interpretado por esses autores como uma estratégia utilizada pelas pacientes para buscarem aproximação e aceitação por parte da figura paterna, dada a distância afetiva percebida entre eles. Dessa maneira, entende-se que é necessário dar atenção às pretensas semelhanças entre pai e filha, inclusive no que se refere à preocupação com a imagem e o peso corporal.

Os resultados encontrados na presente revisão indicam que existe uma proximidade entre a preocupação com alimentação e imagem corporal dos pais e a percebida nas filhas. Isso pode ser interpretado como uma forma de elas buscarem aceitação e utilizarem o pai como modelo. Entretanto, os dados não são conclusivos, o que sugere que esse tema ainda necessita ser abordado com o devido cuidado em estudos futuros, devido à notória escassez de evidências.

Autoestima, depressão e ansiedade. Da mesma forma que as mães das adolescentes e jovens com TAs, os estudos identificaram que os pais também sofrem com baixa autoestima e elevados índices de ansiedade e depressão (Alda, Espina, \& Ortego, 2006; Fassino et al., 2009; Martín et al., 2013). De acordo com Alda et al. (2006), esses sintomas aumentam após o surgimento do TA nas filhas. Os resultados sugerem, portanto, que o desenvolvimento do transtorno das filhas poderia atuar como um gatilho ou, ainda, aumentar os indicadores de funcionamento psicopatológico dos pais.

Tanto os pais como as mães de mulheres com TAs apresentaram níveis elevados de ansiedade e depressão Porém, quando são comparados os dados do casal parental, os resultados são diferentes. Martín et al. (2013) demonstraram que as mães apresentavam níveis mais elevados de sintomas depressivos e ansiosos do que os pais. Somado a isso, os pais percebiam que tinham uma qualidade de vida melhor e se sentiam menos sobrecarregados com o transtorno das filhas do que as mães. Os autores sugerem que uma das explicações para essas diferenças entre pais e mães reside no maior engajamento materno no cuidado das filhas. Além disso, a tendência dos pais à esquiva pode ser entendida como uma maneira de reduzir a sobrecarga desencadeada pelo quadro clínico da filha.

A preocupação com a imagem corporal pode ser um dos fatores que incrementam os níveis de ansiedade e depressão dos pais, assim como pode colaborar com sua baixa autoestima. Entretanto, nenhum dos estudos encontrados teve a intenção de investigar essa correlação, da mesma forma que a maioria deles não teve a intenção de investigar as causas dos sintomas psicopatológicos dos pais. Essa preocupação foi observada com mais destaque nos estudos de Hopper e Dallos (2012), Pilecki e Józefik (2013) e Pilecki, Józefik e Salapa (2014), que destacam a importância de estudar a família de origem e as vivências familiares precoces dos pais como estratégia para melhor compreender seu funcionamento psíquico e, consequentemente, o modo como se aproximam ou se distanciam de suas filhas no presente.

A perspectiva do pai sobre o funcionamento familiar. Dentre as publicações que compõem a presente revisão, apenas três estudos se preocuparam em investigar a família de origem da figura paterna, sondando características de seu funcionamento que poderiam levar ao desenvolvimento de traços psicopatológicos nos pais - esses que, por sua vez, colaborariam com o surgimento dos TAs nas filhas (Hooper \& Dallos, 2012; Pilecki \& Józefik, 2013; Pilecki et al., 2014). Esses estudos investigaram como as experiências vivenciadas pelos pais na família de origem poderiam influenciar a dinâmica das famílias constituídas e o relacionamento estabelecido com as filhas.

Hopper e Dallos (2012) encontraram pais de mulheres com AN que haviam vivenciado momentos de intenso sofrimento na infância. Na análise realizada, os autores concluíram que os pais, defensivamente, com o intuito de amenizar a angústia suscitada pelas experiências de sofrimento, apresentavam uma visão idealizada da família e de sua infância, além de marcado distanciamento em relação à vivência dos afetos. Esses dados podem explicar os achados de Rowa et al. (2001), que indicam que os pais, 
diferentemente das mães e filhas, percebem menor incidência de conflitos familiares. Essa menor sensibilidade paterna às dificuldades emocionais pode ser reflexo da tendência à esquiva, percebida nos pais das mulheres diagnosticadas com TAs. Ao se afastarem dos conflitos, em momentos de maior mobilização emocional, esses pais não conseguem oferecer suporte suficiente às filhas (Hopper \& Dallos, 2012).

Por outro lado, os estudos de Dancyger, Fornari, Scionti, Wisotsky e Sunday (2005) e Pileck e Jósefik (2013) reportam dados diferentes em relação à percepção dos pais sobre o ambiente familiar. Segundo os achados dessas pesquisas, a mãe tende a ver a família como menos conflituosa, enquanto o pai e a filha relataram um ambiente familiar mais disfuncional. Os participantes de Pileck e Jósefik (2013), ao invés de apresentarem idealizações em relação às suas famílias de origem, identificaram-nas como disfuncionais e com poucas condições de oferecer apoio adequado para o desenvolvimento da autonomia dos membros. As filhas mostraram a mesma percepção que os pais sobre suas famílias. Os dados sugerem a importância de os pais manterem contato com um ambiente propício ao desenvolvimento de sua própria autonomia, para que possam oferecer às filhas um suporte facilitador dessa condição.

Independentemente de terem uma percepção idealizada ou não, as evidências fornecidas pelos estudos selecionados denotam a necessidade de se olhar para o funcionamento familiar também a partir da perspectiva do pai. Os dados indicam que o modo como o pai vivencia as relações familiares reflete na maneira como ele se aproxima do cuidado de suas filhas. Quando percebiam a família como menos conflituosa, eles não conseguiam identificar a necessidade de oferecer suporte à filha (Hopper \& Dallos, 2012). Porém, quando identificaram os conflitos familiares, relataram que também não encontraram modelos nas suas famílias de origem que os encorajassem no exercício da paternidade (Pileck \& Józefik, 2013). Nesse sentido, Pilecki et al. (2014) destacaram a importância de se incluir, também, a investigação dos aspectos transgeracionais relacionados à família do pai, a fim de melhor compreender o modo com se estabelecem as relações familiares das pessoas com TAs.

\section{Participação do Pai no Tratamento}

Independentemente da estratégia metodológica adotada pelos autores, todos os artigos analisados apontaram para a importância de se fomentar a investigação dos recursos de personalidade do pai e de se incentivar sua participação no cenário da assistência aos TAs. Apesar dessas recomendações, na presente revisão não foi encontrada qualquer publicação que tenha investigado a assistência prestada por serviços especializados ou programas específicos que promovam sistematicamente a inclusão da figura paterna no tratamento de pacientes com AN e BN. Assim, os estudos analisados sugerem a importância da inclusão do pai no tratamento, mas de fato não assumem esse tema como foco, o que denota a necessidade de se promoverem investigações científicas, no cenário da assistência, dedicadas ao pai das pessoas diagnosticadas com anorexia e bulimia.

Diante das peculiaridades percebidas no funcionamento paterno, principalmente quando se compara ao materno, Martín et al. (2013) sugerem que, na assistência aos TAs, seja realizado um atendimento diferenciado entre pais e mães, visto que apresentam demandas diferenciadas. Entretanto, antes de se proporem essas intervenções, é preciso conhecer quais são as reais necessidades dos pais. $\mathrm{O}$ fato de grande parte dos estudos buscar conhecer quem eles são, partindo apenas da percepção das filhas, aponta para as dificuldades de abordá-los e, consequentemente, de se proporem intervenções que contemplem suas necessidades psicológicas.

\section{CONSIDERAÇÕES FINAIS}

O presente estudo permitiu esquadrinhar o cenário científico atual sobre a figura paterna no âmbito dos principais TAs. O baixo grau de envolvimento e participação dos pais nos cuidados dedicados às suas filhas adoecidas pode ser decorrente do distanciamento e da tendência à esquiva, conforme mencionado nos estudos analisados nesta revisão. Porém, outros fatores podem contribuir para acentuar o baixo envolvimento, como as construções de gênero que determinam o afastamento da figura paterna nos cuidados em geral dos filhos, seja na saúde ou na doença (Vieira \& Nascimento, 2014). Por esse motivo, ressalta-se que, independentemente dos motivos que levam ao distanciamento na relação pai-filha, torna-se necessário prover estudos que permitam compreender a figura paterna a partir da perspectiva do próprio pai.

Acredita-se que tais pesquisas possibilitariam uma compreensão mais ampla da influência da figura paterna tanto no surgimento, como na manutenção dos sintomas de TAs das filhas. Esse conhecimento pode subsidiar o planejamento de intervenções terapêuticas capazes de acolher as necessidades dos pais, potencializando os seus recursos, de modo a capacitá-los a auxiliar suas filhas que estão em situação de sofrimento psíquico.

Os estudos apontam a importância de se incluir o pai no tratamento das filhas com TAs, porém não foram encontradas pesquisas que tenham investigado especificamente como se dá essa participação no contexto da assistência. Por fim, o fato de não ter sido encontrada publicação nacional na área dificulta o desenvolvimento de estratégias de assistência que possam abarcar as demandas específicas dos serviços de saúde na realidade brasileira. Somado a isso, foram observados predomínio de estudos analíticos (quantitativos) e escassez de estudos com escopo compreensivo, o que amplia as possibilidades 
de generalização dos resultados, porém não favorece a análise das especificidades da relação pai-filha. Assim, ressalta-se a necessidade de se investir em pesquisas nacionais e de cunho qualitativo sobre o pai no cenário dos TAs, as quais se preocupem, principalmente, com os aspectos relacionados aos cuidados terapêuticos, como as facilidades e as dificuldades de envolvimento paterno no contexto de tratamento de suas filhas.

\section{REFERÊNCIAS}

Alda, I. O., Espina, A., \& Ortego, M. A. (2006). Un estudio sobre personalidad, ansiedad y depresión en padres de pacientes con un trastorno alimentario. Clínica y Salud, 17(2), 151-170.

American Psychiatric Association. (2013). Diagnostic and statistical manual of mental disorders - fifth edition - DSM-V. Washington, DC: American Psychiatric Association.

Amianto, F., Daga, G. A., Bertorello, A., \& Fassino, S. (2013). Exploring personality clusters among parents of ED subjects: relationship with parents' psychopathology, attachment, and family dynamics. Comprehensive Psychiatry, 54(7), 797-811.

Back, E. A. (2011). Effects of parental relations and upbringing in troubled adolescent eating behaviors. Eating Disorders: The Journal of Treatment \& Prevention, 19(5), 403-424.

Barbieri, V. (2013). Laços e embaraços: O Procedimento de Desenhos de Família com Estórias na compreensão cruzada dos psicodinamismos de mãe e filha. In W. Trinca (Org.), Procedimentos de Desenhos-Estórias (pp. 231-276). São Paulo: Vetor.

Benninghoven, D., Tetsch, N., Kunzendorf, S., \& Jantschek, G. (2007). Perceptual body image of patients with anorexia or bulimia nervosa and their fathers. Eating and Weight Disorders, 12(1), 12-19.

Birman, J. (2006). Arquivos do mal-estar e da resistência. Rio de Janeiro: Civilização Brasileira.

Birman, J. (2007). Laços e desenlaces na contemporaneidade. Jornal de Psicanálise, 40(72), 47-62.

Boarini, M. L. (2003). Refletindo sobre a nova e velha família. Psicologia em Estudo, 8(n. spe), 1-2.

Botta, R. A., \& Dumlao, R. (2002). How do conflict and communication patterns between fathers and daughters contribute to or offset eating disorders?. Health Communication, 14(2), 199-219.

Cruzat, C., Ramírez, P., Melipillán, R., \& Marzolo, P. (2008). Trastornos alimentarios y funcionamiento familiar percibido en una muestra de estudiantes secundarias de la comuna de Concepción, Chile. Psykhe, 17(1), 81-90.

Dancyger, I., Fornari, V., Scionti, L., Wisotsky W., \& Sunday S. (2005). Do daughters with eating disorders agree with their parents' perception of family functioning?. Comprehensive Psychiatry, 46(2), 135-139.

Dixon, R. S., Gill, J. M. W., \& Adair, V. A. (2003). Exploring paternal influences on the dieting behaviors of adolescent girls. Eating Disorders: Journal of Treatment \& Prevention, 11(1), 39-50.

Elliott, J. C. (2010). Fathers, daughters, and anorexia nervosa. Perspectives in Psychiatric Care, 46(1), 37-47.

Enten, R. S., \& Golan, M. (2009). Parenting styles and eating disorder pathology. Appetite, 52(3), 784-787.

Fassino, S., Amianto, F., \& Abbate-Daga, G. (2009). The dynamic relationship of parental personality traits with the personality and psychopathology traits of anorectic and bulimic daughters. Comprehensive Psychiatry, 50(3), 232-239.

Fava, M. V., \& Peres, R. S. (2011). Do vazio mental ao vazio corporal: um olhar psicanalítico sobre as comunidades virtuais pró-anorexia. Paidéia, 21(50), 353-361.

Gaspar, F. L. (2005). A violência do outro na anorexia: Uma problemática de fronteiras. Revista Latinoamericana de Psicopatologia Fundamental, 8(4), 629-643.
Goossens, L., Braet, C., Van Durme, K., Decaluwé, V., \& Bosmans, G. (2012). The parent-child relationship as predictor of eating pathology and weight gain in preadolescents. Journal of Clinical Child Adolescent Psychology, 41(4), 445-457.

Gutzwiller, J., Oliver, J. M., \& Katz, B. M. (2003). Eating dysfunctions in college women: the roles of depression and attachment to fathers. Journal of American College Health, 52(1), 27-32.

Guzmán, M. B., Hitelman, L. J., \& Kaplan, M. D. (2009). Anorexia nervosa: La mirada del padre. Revista Chilena de Psicoanálisis, 26(1), 97-105.

Haycraft, E., \& Blissett, J. (2012). Predictors of paternal and maternal controlling feeding practices with 2- to 5-year-old children. Journal of Nutrition Education and Behavior, 44(5), 390-397.

Henn, C. G., \& Sifuentes, M. (2012). Parentalidade no contexto das necessidades especiais: Revisão sistemática da literatura. Paidéia, 22(51), 131-139.

Hooper, A., \& Dallos, R. (2012). Fathers and daughters: Their relationship and attachment themes in the shadow of an eating disorder. Contemporary Family Therapy, 34(4), 452-467.

Horesh, N., Sommerfeld, E., Wolf, M., Zubery, E., \& Zalsman, G. (2014). Father-daughter relationship and the severity of eating disorders. European Psychiatry: The Journal of the Association of European Psychiatrists, 30(1), 114-120.

Jones, C. J., Harris, G., \& Leung, N. (2005). Parental rearing behaviours and eating disorders: the moderating role of core beliefs. Eating Behaviors, 6(4), 355-364.

Jones, C. J., Leung, N., \& Harris, G. (2006). Father-daughter relationship and eating psychopathology: The mediating role of core beliefs. British Journal of Clinical Psychology, 45(3), 319-330.

Lam, C. B., \& McHale, S. M. (2012). Developmental Patterns and Family Predictors of Adolescent Weight Concerns: A Replication and Extension. International Journal of Eating Disorders, 45(4), 524-530.

Martín, J., Padierna, A., Aguirre, U., González, N., Muñoz, P., \& Quintana, J. M. (2013). Predictors of quality of life and caregiver burden among maternal and paternal caregivers of patients with eating disorders. Psychiatry Research, 210(3), 1107-1115.

McEwen, C., \& Flouri, E. (2009). Fathers' parenting, adverse life events, and adolescents' emotional and eating disorder symptoms: the role of emotion regulation. European Child \& Adolescent Psychiatry, 18(4), 206-216.

Minayo, M. C. S. (2007). O desafio do conhecimento: pesquisa qualitativa em saúde ( $9^{\mathrm{a}}$ ed.). São Paulo/Rio de Janeiro: Hucitec/ABRASCO.

Miranda, M. R. (2010). A representação simbólica nas perturbações alimentares à luz da complexidade da relação mãe-filha. In A. P. Gonzaga, \& C. Weinberg (Orgs.), Psicanálise de transtornos alimentares. São Paulo: Primavera Editorial.

MOURA, F. E. G. A., Santos, M. A., RIBEIRO, R. P. P. (2015). A constituição da relação mãe-filha e o desenvolvimento dos transtornos alimentares. Estudos de Psicologia (PUCCAMP), $32,233-247$.

Nodin, N., \& Leal, I. P. (2005). Representações paternas na anorexia nervosa. Análise Psicológica, 23(2), 201-208.

Oliveira, E. A., \& Santos, M. A. (2006). Perfil psicológico de pacientes com anorexia e bulimia nervosas: a ótica do psicodiagnóstico. Medicina, 39(3), 353-360. 
Oliveira, A. G., \& Silva, R. R. (2011). Pai contemporâneo: Diálogos entre pesquisadores brasileiros no período de 1998 a 2008. Psicologia Argumento, 29(66), 353-360.

Pace, U., Cacioppo, M., \& Schimmenti, A. (2012). The moderating role of father's care on the onset of binge eating symptoms among female late adolescents with insecure attachment. Child Psychiatry Human Development, 43(2), 282-292.

Pilecki, M. W., \& Józefik, B. (2013). Perception of transgenerational family relationships: comparison of eating-disordered patients and their parents. Medical Science Monitor, 19, 1114-1124.

Pilecki, M. W., Józefik, B., \& Salapa, K. (2014). Correlations between the perception of family functioning by patients with eating disorders and their parents and the perception of relations in the parents' families of origin. Psychiatria Polska, 48(4), 789-808.

Rowa, K., Kerig, P. K., \& Geller, J. (2001). The family and anorexia nervosa: examining parent-child boundary problems. European Eating Disorders Review, 9(2), 97-114.

Santos, A. F., \& Santos, M. A. (2015). Estresse e burnout no trabalho em Oncologia Pediátrica: Revisão integrativa da literatura. Psicologia: Ciência e Profissão, 35(2), 437-456.
Santos, M. A., Garcia, R. W. D., \& Santos, M. L. (2015). A sujeição aos padrões corporais culturalmente construídos em mulheres de baixa renda. Demetra: Alimentação, Nutrição e Saúde, 10(4), 761-774.

Souza, L.V., \& Santos, M. A. (2007). Grupo de familiares de pessoas com transtornos alimentares: Um espaço co-construído. Vinculo, 4, 38-47.

Smink, F. R., Van Hoeken, D., \& Hoek, H. W. (2013). Epidemiology, course, and outcome of eating disorders. Current Opinion in Psychiatry, 26(6), 543-548.

Valdanha, E. D., Scorsolini-Comin, F., Peres, R. S., \& Santos, M. A. (2013). Influência familiar na anorexia nervosa: em busca das melhores evidências científicas. Jornal Brasileiro de Psiquiatria, 62(3), 225-233.

Vieira, G. T., \& Nascimento, A. R. A. (2014). Aspectos psicossociais da construção da identidade paterna. Psicologia: Teoria e Prática, 16(1), 57-68.

Whitemore, R., \& Knafl, K. (2005). The integrative review: updated methodology. Journal of Advanced Nursing, 52(5), 546-553.

Zimerman, D. E. (1999). Fundamentos psicanaliticos: teoria, técnica e clínica, uma abordagem didática. Porto Alegre, RS: Artes Médicas. 